\title{
Comparison of BISAP, Ranson, MCTSI, and APACHE II in Predicting Severity and Prognoses of Hyperlipidemic Acute Pancreatitis in Chinese Patients
}

\author{
Lixin Yang, ${ }^{1}$ Jing Liu, ${ }^{2}$ Yun Xing, ${ }^{1}$ Lichuan Du, ${ }^{1}$ Jing Chen, ${ }^{1}$ Xin Liu, ${ }^{3}$ and Jianyu Hao ${ }^{1}$ \\ ${ }^{1}$ Department of Gastroenterology, Beijing Chaoyang Hospital, Capital Medical University, Beijing 100020, China \\ ${ }^{2}$ Beijing First Hospital of Integrated Chinese and Western Medicine, Beijing, China \\ ${ }^{3}$ Hebei Xianghe People's Hospital, Xianghe, China
}

Correspondence should be addressed to Lixin Yang; cyyyxhk@163.com

Received 14 July 2016; Revised 24 September 2016; Accepted 9 October 2016

Academic Editor: Atsushi Irisawa

Copyright (C) 2016 Lixin Yang et al. This is an open access article distributed under the Creative Commons Attribution License, which permits unrestricted use, distribution, and reproduction in any medium, provided the original work is properly cited.

In recent years, with the developing of living standard, hyperlipidemia becomes the second major reason of acute pancreatitis. It is important to predict the severity and prognosis at early stage of hyperlipidemic acute pancreatitis (HLAP). We compared the BISAP, Ranson, MCTSI, and APACHE II scoring system in predicting MSAP and SAP, local complications, and mortality of HLAP. A total of 326 diagnosed hyperlipidemic acute pancreatitis patients from August 2006 to July 2015 were studied retrospectively. Our result showed that all four scoring systems can be used to predict the severity, local complications, and mortality of HLAP. Ranson did not have significant advantage in predicting severity and prognosis of HLAP compared to other three scoring systems. APACHE II was the best in predicting severity of HLAP, but it had shortcoming in predicting local complications. MCTSI had outstanding performance in predicting local complications, but it was poor in predicting severity and mortality. BISAP score had high accuracy in assessment of severity, local complications, and mortality of HLAP, but the accuracy still needs to be improved in the future.

\section{Introduction}

Recently, with the change of people's living condition and life style, hyperlipidemia comes to the second major reason of acute pancreatitis. A study involving 2416 cases diagnosed with acute pancreatitis (AP) from 2006 to 2010 in Beijing found that $255(10.36 \%)$ cases were hyperlipidemic acute pancreatitis (HLAP) [1]. An analysis carried by $\mathrm{Xu}$ et al. claims that, in the period of 2012 to 2014, HLAP accounted for $19.1 \%$ of total AP [2]. Compared with NHLAP, HLAP are characterized by critical condition and high recurrence rate [3]. So, it is important to predict the severity and prognosis of HLAP at early stage, which is beneficial for individualized treatment and prognosis.

There are four frequently used scoring systems of AP, including BISAP (bedside index for severity in acute pancreatitis), Ranson score, MCTSI (modified CT severity index), and APACHE II (acute physiology and chronic health evaluation scoring system). To our knowledge, there is no largepopulation-based study in assessment of severity and prog- nosis of HLAP. In this paper, a total of 326 cases diagnosed with HLAP from 2007 to 2015 admitted to a single center were retrospectively analyzed to compare the prediction value of four scoring systems.

\section{Material and Method}

2.1. Data. We retrospectively analyzed a series of 326 patients diagnosed with HALP who were admitted to Beijing ChaoYang Hospital, Capital Medical University, in a period of August 2007 to July 2015 (184 males, 142 females; age ranging from 14 to 85; mean age of 44 years). Of the 326 patients included, 65 (19.9\%) had moderately severe acute pancreatitis (MSAP), 27 (8.3\%) had severe acute pancreatitis (SAP), 28 (8.59\%) had local complications, and 9 died $(2.8 \%)$.

Local complications include acute peripancreatic fluid collection, pancreatic pseudocyst, walled-off necrosis, infected necrosis, pleural effusion, intestinal fistula, and pancreatic pseudocyst hemorrhage. Of the 28 patients with local complications, 21 had two or more local complications. 72 
TABLE 1: Analysis of the four scoring systems in predicting severity of HLAP.

\begin{tabular}{|c|c|c|c|c|c|c|}
\hline $\begin{array}{l}\text { Scoring system } \\
\text {. }\end{array}$ & MSAP and SAP & MAP & Total & OR & $\chi^{2}$ & $p$ \\
\hline \multicolumn{7}{|l|}{ BISAP } \\
\hline$\geq 3$ & 48 & 23 & 71 & \multirow[t]{2}{*}{10.0} & \multirow[t]{2}{*}{73.1} & \multirow[t]{2}{*}{$<0.05$} \\
\hline$<3$ & 44 & 211 & 255 & & & \\
\hline \multicolumn{7}{|l|}{ Ranson } \\
\hline$\geq 3$ & 43 & 37 & 80 & \multirow[t]{2}{*}{4.7} & \multirow[t]{2}{*}{34.1} & \multirow[t]{2}{*}{$<0.05$} \\
\hline$<3$ & 49 & 197 & 246 & & & \\
\hline \multicolumn{7}{|l|}{ APACHE II } \\
\hline$\geq 8$ & 52 & 26 & 78 & \multirow[t]{2}{*}{10.4} & \multirow[t]{2}{*}{74.8} & \multirow[t]{2}{*}{$<0.05$} \\
\hline$<8$ & 40 & 208 & 248 & & & \\
\hline \multicolumn{7}{|l|}{ MCTSI } \\
\hline$\geq 4$ & 33 & 17 & 50 & \multirow[t]{2}{*}{8.2} & \multirow[t]{2}{*}{45.6} & \multirow[t]{2}{*}{$<0.05$} \\
\hline$<4$ & 59 & 217 & 276 & & & \\
\hline \multicolumn{7}{|c|}{ MAP $=$ mild acute pancreatitis } \\
\hline
\end{tabular}

patients had a relapse, and the recurrence rate is $22.09 \%$. Among them $15(7.67 \%)$ had 3 or more recurrent relapses.

2.2. Diagnostic Criteria and Scoring System. The diagnostic criteria of acute pancreatitis referred to Chinese guideline for diagnosis and treatment of acute pancreatitis and Atlanta Classification of Acute Pancreatitis [4, 5].

The diagnosis of acute pancreatitis, whether in the presence or absence of underlying chronic pancreatitis, requires two of the following three features: (1) abdominal pain suggestive strongly of acute pancreatitis, (2) serum amylase and/or lipase activity at least 3 times greater than the upper limit of normal, and (3) characteristic findings of acute pancreatitis on transabdominal ultrasonography, contrastenhanced ECT, or magnetic resonance imaging (MRI).

The grading of severity of AP referred to Chinese guideline for diagnosis and treatment of acute pancreatitis as follows:

(1) MAP meets AP diagnostic criteria. MAP requires one of the following three features: no organ failure; no local or systemic complications; and Ranson score $<3$ points; APACHE II score $<8$; BISAP score $<3$ points; and MCTSI score $<4$ points.

(2) MSAP meets AP diagnostic criteria. At the same time, MSAP should meet one of the following conditions: (1) Ranson score $\geq 3$ points; (2) APACHE score $\geq$ 8 points; (3) BISAP score $\geq 3$ points; (4) MCTSI $\geq 4$ points; (5) transient organ failure (<48 h); (6) pseudocyst, pancreatic fistula, or pancreatic abscess that needs surgical operation.

(3) SAP meets AP diagnostic criteria and have the presence of persistent $(>48 \mathrm{~h}$ ) organ failure (single or multiple organ) and the modified Marshall score $\geq 2$ points.

HLAP was diagnosed if serum triglycerides (TG) reached $11.3 \mathrm{mmol} / \mathrm{L}$, or TG was more than 5.56 to $11.3 \mathrm{mmol} / \mathrm{L}$ accompanied by chylemia, excluding other etiologies of AP (gallstone, drug, infection, etc.) $[6,7]$.

BISAP and APACHE II score were calculated in 24 hours after admission. Ranson score was calculated in 48 hours. MCTSI score was calculated in patients with contrastenhanced CT scan within 3 days of onset. The score is calculated based on the worst value of each criteria [8-10].

Local complications were evaluated by contrast-enhanced computed tomography (CECT). CECT scans were retrospectively and independently reviewed by two experienced abdominal radiologists who were unaware of presenting signs and symptoms or of patient outcomes. Kappa statistic was calculated for measuring agreement between two radiologists and the result indicates good agreement. We obtained approval from Institutional Review Board of Beijing ChaoYang Hospital for this study.

2.3. Statistical Analysis. The data was statistically analyzed using SPSS software 19.0. BISAP, Ranson, MCTSI, and APACHE II were compared in predicting severity, location complications, and mortality of HLAP, using chi-square testing, Fisher's exact probability test, and receiver operating characteristic curve. Odds ratio (OR), sensitivity, specificity, positive predictive value (PPV), positive likelihood ratio (PLR), Youden index, and area under ROC curve (AUC) were calculated. A $p$ value $<0.05$ was considered as statistically significant.

\section{Result}

3.1. Predicting Value of Four Scoring Systems in HLAP. Tables 1,2 , and 3 show that the incidence of MSAP and SAP, local complications, and mortality in patients with BISAP score $\geq$ 3 , Ranson score $\geq 3$, APACHE II score $\geq 8$, and MCTSI score $\geq 4$ were significantly higher than those in BISAP score $<3$, Ranson score $<3$, APACHE II score $<8$, and MCTSI $<4(p<$ $0.05)$. 
TABLE 2: Analysis of the four scoring systems in predicting location complications of HLAP.

\begin{tabular}{|c|c|c|c|c|c|c|}
\hline Scoring system & Local complication & No local complication & Total & OR & $\chi^{2}$ & $p$ \\
\hline \multicolumn{7}{|l|}{ BISAP } \\
\hline$\geq 3$ & 15 & 56 & 71 & 5.0 & 18.2 & $<0.05$ \\
\hline$<3$ & 13 & 242 & 255 & & & \\
\hline \multicolumn{7}{|l|}{ Ranson } \\
\hline$\geq 3$ & 16 & 64 & 80 & 4.9 & 17.6 & $<0.05$ \\
\hline$<3$ & 12 & 234 & 246 & & & \\
\hline \multicolumn{7}{|l|}{ APACHE II } \\
\hline$\geq 8$ & 12 & 66 & 78 & 2.6 & 6.0 & $<0.05$ \\
\hline$<8$ & 16 & 232 & 248 & & & \\
\hline \multicolumn{7}{|l|}{ MCTSI } \\
\hline$\geq 4$ & 19 & 31 & 50 & 18.2 & 65.1 & $<0.05$ \\
\hline$<4$ & 9 & 267 & 276 & & & \\
\hline
\end{tabular}

TABLE 3: Analysis of the four scoring systems in predicting mortality of HLAP.

\begin{tabular}{|c|c|c|c|c|c|c|}
\hline Scoring system & Mortality & Survival & Total & OR & $x^{2}$ & $p$ \\
\hline \multicolumn{7}{|l|}{ BISAP } \\
\hline$\geq 3$ & 8 & 63 & 71 & 32.3 & 24.5 & $<0.05$ \\
\hline$<3$ & 1 & 254 & 255 & & & \\
\hline \multicolumn{7}{|l|}{ Ranson } \\
\hline$\geq 3$ & 7 & 73 & 80 & 11.7 & 14.2 & $<0.05$ \\
\hline$<3$ & 2 & 242 & 246 & & & \\
\hline \multicolumn{7}{|l|}{ APACHE II } \\
\hline$\geq 8$ & 8 & 70 & 78 & 28.2 & 21.5 & $<0.05$ \\
\hline$<8$ & 1 & 247 & 248 & & & \\
\hline \multicolumn{7}{|l|}{ MCTSI } \\
\hline$\geq 4$ & 7 & 43 & 50 & 21.7 & 27.1 & $<0.05$ \\
\hline$<4$ & 2 & 275 & 276 & & & \\
\hline
\end{tabular}

3.2. Comparison of the Four Scoring Systems in Predicting Severity of HLAP. Table 4 and Figure 1(a) show the sensitivity, specificity, PPV, PLR, Youden index, and AUC of BISAP, Ranson, MCTSI, and APACHE II in predicting severity of HLAP. In assessment of severity, the sensitivity and AUC of APACHE II were $57 \%$ and 0.814 , which were the highest. BISAP was second with sensitivity of $54 \%$. And the AUC of BISAP was 0.795 .

3.3. Comparison of the Four Scoring Systems in Predicting Local Complications of HLAP. Table 4 and Figure 1(b) show the sensitivity, specificity, PPV, PLR, Youden index, and AUC of BISAP, Ranson, MCTSI, and APACHE II in predicting location complications of HLAP. In assessment of local complications, the sensitivity and AUC of MCTSI were $68 \%$ and 0.791 , which were the highest. BISAP was second with AUC of 0.731 .

3.4. Comparison of the Four Scoring Systems in Predicting Mortality of HLAP. Table 4 and Figure 1(c) show the sensitivity, specificity, PPV, PLR, Youden index, and AUC of BISAP, Ranson, MCTSI, and APACHE II in assessment of mortality of HLAP. In assessment of mortality, the sensitivity and AUC of BISAP were $89 \%$ and 0.867 , which were the highest. The second was APACHE II with AUC of 0.854 . Both APACHE II and BISAP had the highest sensitivity of $89 \%$.

\section{Discussion}

Compared with NHLAP, HLAP has the following features: (1) high recurrence rate: the higher the blood lipid level is, the greater the possibility of recurrence; serum TG lower than $5.56 \mathrm{mmol} / \mathrm{L}$ can prevent episodes of pancreatitis; (2) serum TG above $11.3 \mathrm{mmol} / \mathrm{L}$; (3) xanthomata in the limbs, buttocks and back, retinal lipemia, hepatosplenomegaly, and fatty liver which can be found in patients with severe hypertriglyceridemia (HTG) because of lipid deposition; (4) patients with HLAP having younger age of onset. Uncontrolled diabetes, obesity, alcoholism, pregnancy, family history of hyperlipidemia are thought to be the risk factors for HLAP $[11,12]$.

It has been reported that the incidence of sever acute pancreatitis (SAP) and organ dysfunction, recurrence rate, and mortality of HLAP were significantly higher than those of 
TABLE 4: Comparison of 4 scoring systems in predicting severity, local complications, and mortality of HLAP.

\begin{tabular}{|c|c|c|c|c|c|c|c|}
\hline Scoring system & Sensitivity & Specificity & PPV (\%) & PLR & Youden index & AUC & $95 \% \mathrm{CI}$ \\
\hline \multicolumn{8}{|l|}{$M S A P$ and $S A P$} \\
\hline BISAP & 54 & 86 & 68 & 3.9 & 0.4 & 0.795 & $0.660-0.929$ \\
\hline Ranson & 46 & 84 & 54 & 2.9 & 0.3 & 0.766 & $0.616-0.916$ \\
\hline APACHE II & 57 & 89 & 67 & 5.2 & 0.46 & 0.814 & $0.686-0.942$ \\
\hline MCTSI & 36 & 94 & 66 & 6 & 0.3 & 0.654 & $0.492-0.815$ \\
\hline \multicolumn{8}{|c|}{ Location complications } \\
\hline BISAP & 54 & 81 & 21 & 2.8 & 0.35 & 0.731 & $0.581-0.881$ \\
\hline Ranson & 57 & 79 & 20 & 2.7 & 0.36 & 0.698 & $0.532-0.865$ \\
\hline APACHE II & 43 & 78 & 15 & 2.0 & 0.21 & 0.580 & $0.407-0.753$ \\
\hline MCTSI & 68 & 90 & 38 & 6.8 & 0.58 & 0.791 & $0.656-0.925$ \\
\hline \multicolumn{8}{|l|}{ Mortality } \\
\hline BISAP & 89 & 80 & 15 & 4.5 & 0.69 & 0.867 & $0.758-0.976$ \\
\hline Ranson & 78 & 77 & 9 & 3.4 & 0.55 & 0.842 & $0.722-0.962$ \\
\hline APACHE II & 89 & 78 & 10 & 4.0 & 0.67 & 0.854 & $0.724-0.984$ \\
\hline MCTSI & 78 & 86 & 14 & 5.6 & 0.64 & 0.839 & $0.721-0.957$ \\
\hline
\end{tabular}

acute biliary pancreatitis [3]. So, it is extremely important to predict and evaluate the severity of HLAP at early stage. The clinical scoring system is a practical tool for the doctor to find potential patients who need intensive care. However, there is little research on this aspect. A total of 129 cases of HLAP show that it is fairly accurate to predict SAP, complications, organ failure, and prognostic of HLAP, using BISAP, Ranson, SIRS, and MCTSI (area under the curve ranges from 0.938 to $0.668)[13,14]$.

BISAP, Ranson, APACHE II, and MCTSI are the most commonly used scoring systems to evaluate acute pancreatitis, but they still have limitations. Firstly, APACHE II scoring system is not convenient to operate, because it has too many parameters to collect. Secondly, different scoring systems contain common parameters; for example, SIRS is a composite parameter used in both BISAP and APACHE II. However, different scoring systems have different way to evaluate one parameter. Taking blood urea nitrogen (BUN) as an example, Ranson takes the increasing level of BUN as criteria, but BISAP takes the absolute value as the criteria. So it takes a lot of time using variety of scoring systems to predict the prognosis.

We hope to select one scoring system that can predict the prognosis of HLAP accurately and easily. So we compared the accuracy of four scoring systems in predicting severity, complications, and mortality.

Our study showed that the incidence of MSAP and SAP, local complications, and mortality were significantly higher in BISAP score $\geq 3$, Ranson score $\geq 3$, APACHE II score $\geq$ 8 , and MCTSI score $\geq 4$ than in BISAP score $<3$, Ranson score $<3$, APACHE II score $<8$, and MCTSI $<4(p<0.05)$. Therefore, all four scoring systems can be used to predict the severity, local complications, and mortality of HLAP.

In 1974, Ranson et al. selected 11 indicators associated with the severity of AP by screening 43 clinical and biochemical indicators [8]. Our study showed that Ranson score was poor in predicting severity and prognosis of HLAP. The AUC of it ranked the third in every aspect we considered. In assessment of MSAP and SAP and mortality, the AUC of Ranson was higher than MCTSI. And Ranson had the lowest positive predictive value, positive likelihood ratio, and Youden index compared with other scoring systems. In assessment of local complications, Ranson was only better than APACHE II.

APACHE II is a frequently used scoring system to assess severity of AP. It consists of three parts, namely, acute physiology score, age, and chronic health score. Our study showed that the APACHE II had highest accuracy in predicting MSAP and SAP and did a good job in predicting mortality. But APACHE II was poor in assessment of local complications. In assessment of MSAP and SAP, the AUC and Youden index of APACHE II were 0.814 and 0.46 , respectively, which were the highest among these four scoring systems. In assessment of mortality, it had the second highest AUC (0.854) and Youden index (0.67), lower than BISAP. In terms of assessing local complications, APACHE II had the lowest AUC (0.580), PPV (15), PLR (2.0), and Youden index (0.21).

MCTSI is a clinical radiological imaging scoring system for evaluating the mortality and local complications of AP. Contrast-enhanced CT is the gold standard for diagnosis of necrotizing pancreatitis and acute peripancreatic fluid collection [10]. In our study, MCTSI had outstanding performance in predicting location complications, with AUC of 0.791 . However, it was poor in predicting severity and mortality. The sensitivity and AUC of MCTSI in assessment of MSAP and SAP and mortality were $36 \%, 0.654$ and $78 \%, 0.839$, respectively, which were lower than the other three scoring systems. According to the retrospective study by Bollen et al., there were no significant differences in prediction accuracies between $7 \mathrm{CT}$ scoring systems and two clinical scoring systems (APACHE II, BISAP), so contrast-enhanced CT scan was not recommended for severity assessment on admission [15]. That conforms to our study.

BISAP was proposed to construct a simple and accurate clinical scoring system to estimate the mortality risk of AP 


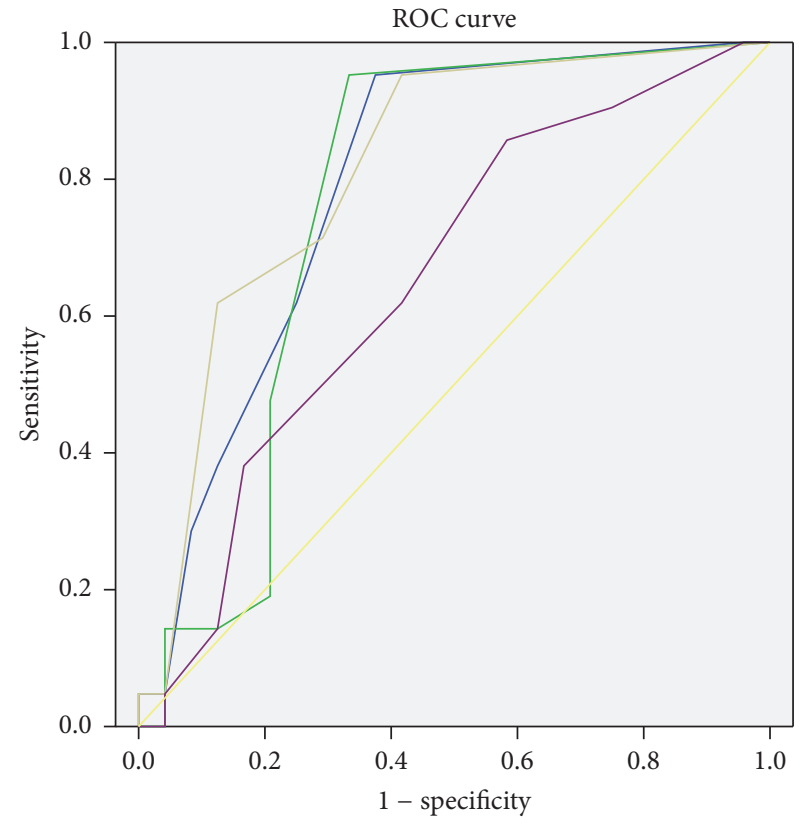

Source of the curve

$\begin{array}{ll}- \text { BISAP } & - \text { MCTSI } \\ \text { Ranson } & \text { Reference line } \\ \text { APACHE II } & \end{array}$

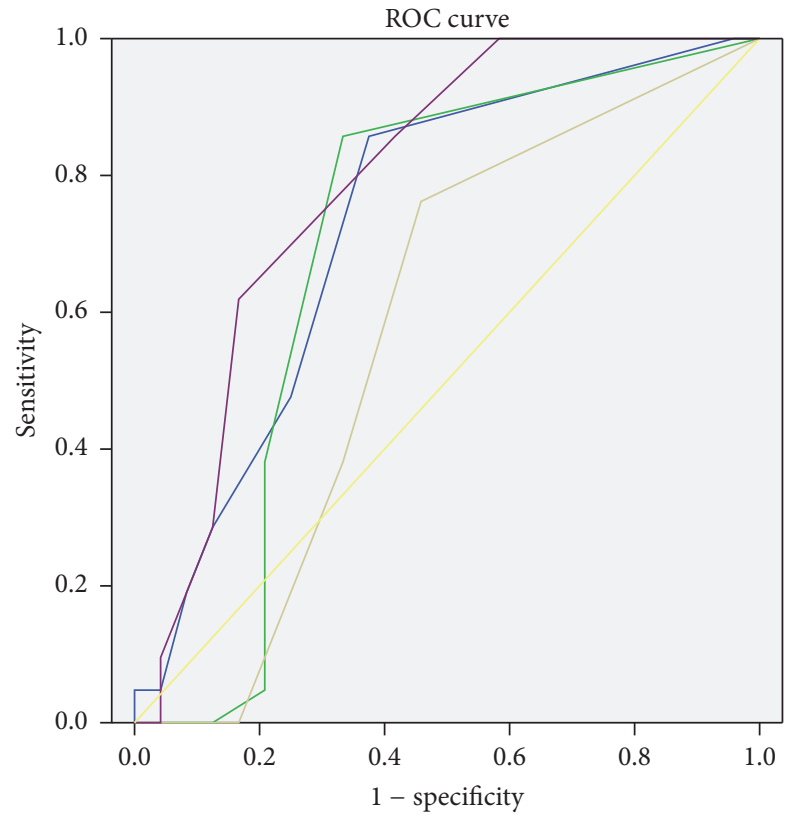

Source of the curve

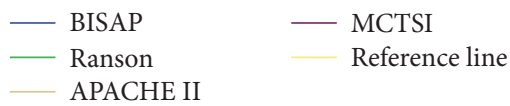

(a)

(b)

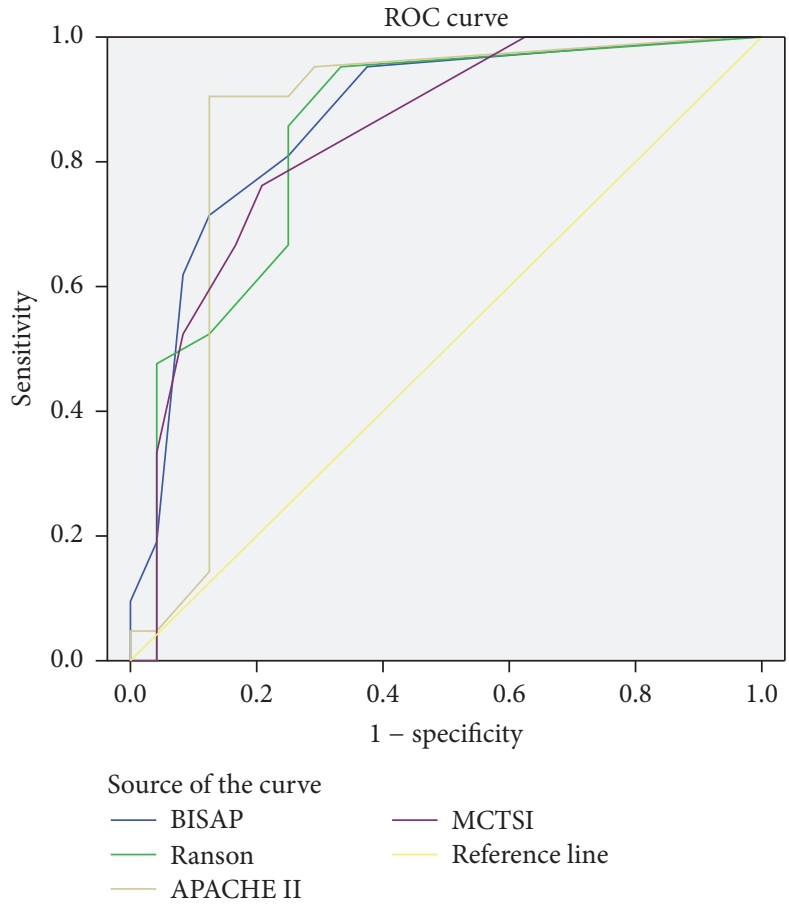

(c)

FIGURE 1: ROC curve of 4 scoring systems in predicting severity, local complications, and mortality of HLAP. (a) ROC curve of 4 scoring systems in predicting severity of HLAP. (b) ROC curve of 4 scoring systems in predicting local complications of HLAP. (c) ROC curve of 4 scoring systems in predicting mortality of HLAP. 
at early stage. BISAP analyzed the in-hospital mortality risk of AP using classification and regression tree analysis, and finally five variables were selected. This scoring system was established from a study involving 17992 cases diagnosed with AP from 212 hospitals. Compared with the APACHE II, the validity of the BISAP score was confirmed using a data of 18256 cases diagnosed with AP from 177 hospitals [9]. Singh et al. applied BISAP to evaluate the severity and mortality in a prospective study enrolling 397 cases diagnosed with AP and came to the same conclusion that BISAP was effective to evaluate the severity of AP [16]. A study by Papachristou et al. indicated that the sensitivity and specificity of BISAP were not worse than "traditional" scoring system (Ranson score, APACHE II, and MCTSI) [17]. In our study, BISAP had high accuracy in predicting MSAP and SAP, local complications, and mortality. In predicting mortality of HLAP, the sensitivity (89\%), Youden index (0.69), and AUC (0.867) were the highest in all scoring systems. In predicting MSAP and SAP, the AUC of BISAP was slightly lower than APACHE II. In predicting local complications, the AUC of BISAP was slightly lower than MCTSI. So, BISAP performed better than other three scoring systems in every aspect we compared in this study.

In conclusion, Ranson did not have significant advantage in predicting severity and prognosis of HLAP. APACHE II was the best in predicting severity, but it had shortcoming in predicting local complications. MCTSI had outstanding performance in predicting local complications. But it is poor in predicting severity and mortality. BISAP score had high accuracy in predicting severity, local complications, and mortality of HLAP. And BISAP score has the advantages of having less parameters and being easy to operate. Further studies in comparison to these four scoring systems appear to be needed, since our study was a single-center study. Nevertheless, we recommended using BISAP to predict the severity and prognosis of HLAP.

However, the accuracy of BISAP should be promoted in further study. In assessment of severity, for example, the AUC value of APACHE II was the highest (0.814), while BISAP was slightly lower (0.795). Mounzer et al. had shown that the current scoring system for acute pancreatitis had reached the maximum efficacy in prediction of organ failure. It was more accurate to predict the severity of combined use of several scoring systems, but it was inconvenient for clinical practice. Unless new scoring system was proposed, it was difficult to improve the prediction accuracy of AP [18].

Another study showed that, compared with NHLAP, the C-reaction protein (CRP) in patients with HLAP was significantly higher on days $1,2,3,4$, and 6 . That means CRP has predictive value in patients with HLAP $[13,14]$. We believed that the diagnostic accuracy will be improved by combining the scoring systems with biochemical parameters correlating with the severity of HLAP, such as CRP and high density lipoprotein. And that is the direction of our further studies $[13,19]$.

\section{Competing Interests}

All authors declare no competing financial interests.

\section{Authors' Contributions}

Lixin Yang designed the work that led to the submission, played an important role in interpreting the results, and wrote the manuscript. Jing Liu participated in the design of the study and contributed to analysis and manuscript preparation. Yun Xing participated in the design of the study and data acquisition and revised the manuscript. Lichuan $\mathrm{Du}$ carried out sample processing and helped perform the analysis with constructive discussions. Jing Chen helped perform the analysis with constructive discussions and compiled the manuscript. Xin Liu helped perform the analysis with constructive discussions. Jianyu Hao contributed to the conception of the study and participated in the design of the study. All authors reviewed the manuscript.

\section{References}

[1] Y. Zheng, Z. Zhou, H. Li et al., "A multicenter study on etiology of acute pancreatitis in Beijing during 5 years," Journal of the Pancreas, vol. 44, no. 3, pp. 409-414, 2015.

[2] H. Xu, Y. Li, J. Yan et al., "Severity analyese of acute pancreatitis based on etiology," Zhonghua Yi Xue Za Zhi, vol. 94, no. 41, pp. 3220-3223, 2014.

[3] Y.-X. Huang, L. Jia, S.-M. Jiang, S.-B. Wang, M.-X. Li, and B.-H. Yang, "Incidence and clinical features of hyperlipidemic acute pancreatitis from Guangdong, China: a retrospective multicenter study," Pancreas, vol. 43, no. 4, pp. 548-552, 2014.

[4] Pancreatology Group, Gastroenterology Society, Chinese Medical Association, Editorial Committee of Chinese Journal of Pancreatology, and Editorial Committee of Chinese Journal of Digestion, "Guide for treatment of acute pancreatitis in China," Chinese Journal of Digestion, vol. 13, no. 2, pp. 73-78, 2013.

[5] Acute Pancreatitis Classification Working Group, "Revision of the Atlanta classification of acute pancreatitis," Documents/Atlanta Classification, 2008.

[6] D. Yadav and C. S. Pitchumoni, "Pancreatic and biliary diseases: issues in hyperlipidemic pancreatitis," Journal of Clinical Gastroenterology, vol. 36, no. 1, pp. 54-62, 2003.

[7] J. Scherer, V. P. Singh, C. S. Pitchumoni, and D. Yadav, "Issues in hypertriglyceridemic pancreatitis: an update," Journal of Clinical Gastroenterology, vol. 48, no. 3, pp. 195-203, 2014.

[8] J. H. Ranson, K. M. Rifkind, D. F. Roses, S. D. Fink, K. Eng, and F. C. Spencer, "Prognostic signs and the role of operative management in acute pancreatitis," Surgery Gynecology and Obstetrics, vol. 139, no. 1, pp. 69-81, 1974.

[9] B. U. Wu, R. S. Johannes, X. Sun, Y. Tabak, D. L. Conwell, and P. A. Banks, "The early prediction of mortality in acute pancreatitis: a large population-based study," Gut, vol. 57, no. 12, pp. 1698-1703, 2008.

[10] K. J. Mortele, W. Wiesner, L. Intriere et al., "A modified CT severity index for evaluating acute pancreatitis: improved correlation with patient outcome," American Journal of Roentgenology, vol. 183, no. 5, pp. 1261-1265, 2004.

[11] W. Tsuang, U. Navaneethan, L. Ruiz, J. B. Palascak, and A. Gelrud, "Hypertriglyceridemic pancreatitis: presentation and management," The American Journal of Gastroenterology, vol. 104, no. 4, pp. 984-991, 2009.

[12] P. Valdivielso, A. Ramírez-Bueno, and N. Ewald, "Current knowledge of hypertriglyceridemic pancreatitis," European Journal of Internal Medicine, vol. 25, no. 8, pp. 689-694, 2014. 
[13] L. Qiu, R. Q. Sun, R. R. Jia et al., "Comparison of existing clinical scoring systems in predicting severity and prognoses of hyperlipidemic acute pancreatitis in chinese patients: A Retrospective Study," Medicine, vol. 94, no. 23, p. e957, 2015.

[14] G. Yin, G. Hu, X. Cang et al., "C-reactive protein: rethinking its role in evaluating the severity of hyperlipidemic acute pancreatitis," Pancreas, vol. 43, no. 8, pp. 1323-1328, 2014.

[15] T. L. Bollen, V. K. Singh, R. Maurer et al., "A comparative evaluation of radiologic and clinical scoring systems in the early prediction of severity in acute pancreatitis," The American Journal of Gastroenterology, vol. 107, no. 4, pp. 612-619, 2012.

[16] V. K. Singh, B. U. Wu, T. L. Bollen et al., "A prospective evaluation of the bedside index for severity in acute pancreatitis score in assessing mortality and intermediate markers of severity in acute pancreatitis," American Journal of Gastroenterology, vol. 104, no. 4, pp. 966-971, 2009.

[17] G. I. Papachristou, V. Muddana, D. Yadav et al., "Comparison of BISAP, Ranson's, APACHE-II, and CTSI scores in predicting organ failure, complications, and mortality in acute pancreatitis," American Journal of Gastroenterology, vol. 105, no. 2, pp. 435-441, 2010.

[18] R. Mounzer, C. J. Langmead, B. U. Wu et al., "Comparison of existing clinical scoring systems to predict persistent organ failure in patients with acute pancreatitis," Gastroenterology, vol. 142, no. 7, pp. 1476-1482, 2012.

[19] M. S. Bugdaci, M. Sokmen, S. S. Zuhur, and Y. Altuntas, "Lipid profile changes and importance of low serum $\alpha$-lipoprotein fraction (high-density lipoprotein) in cases with acute pancreatitis," Pancreas, vol. 40, no. 8, pp. 1241-1244, 2011. 


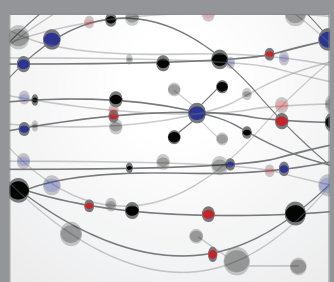

The Scientific World Journal
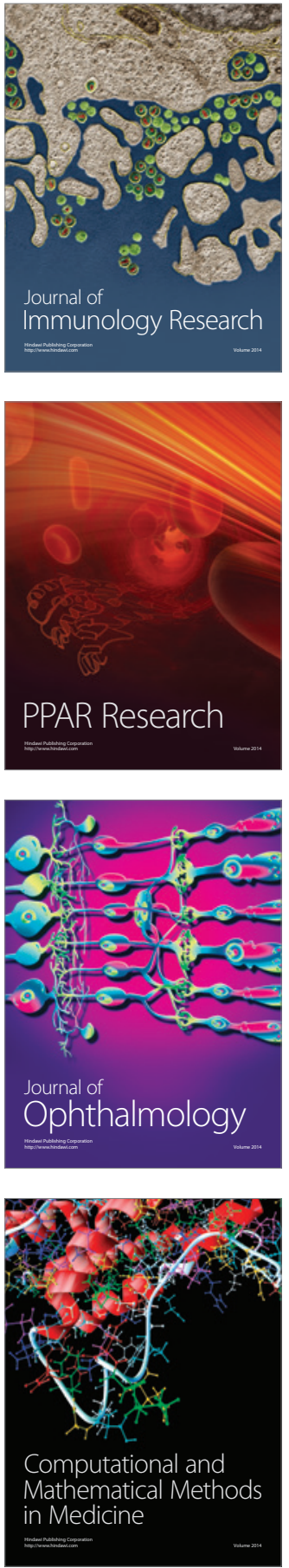

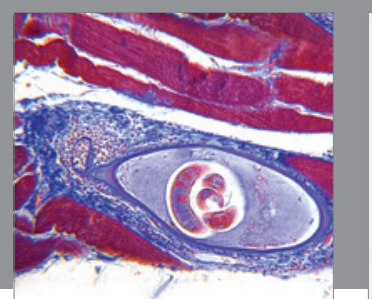

Gastroenterology Research and Practice

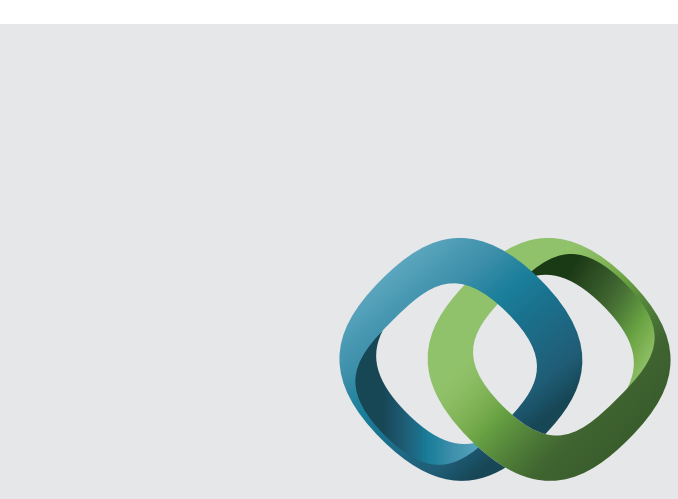

\section{Hindawi}

Submit your manuscripts at

http://www.hindawi.com
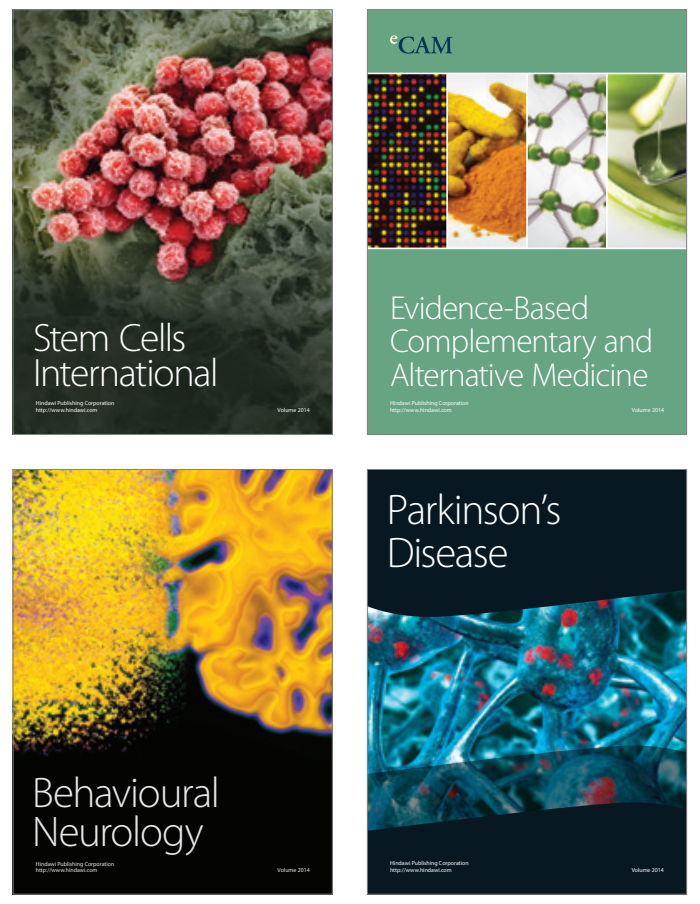
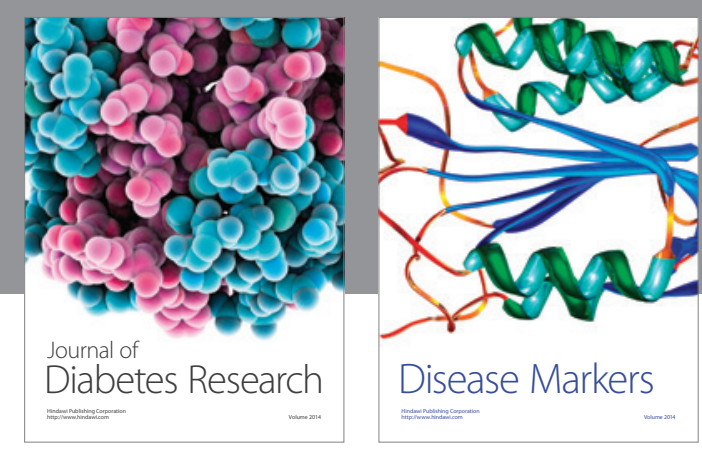

Disease Markers
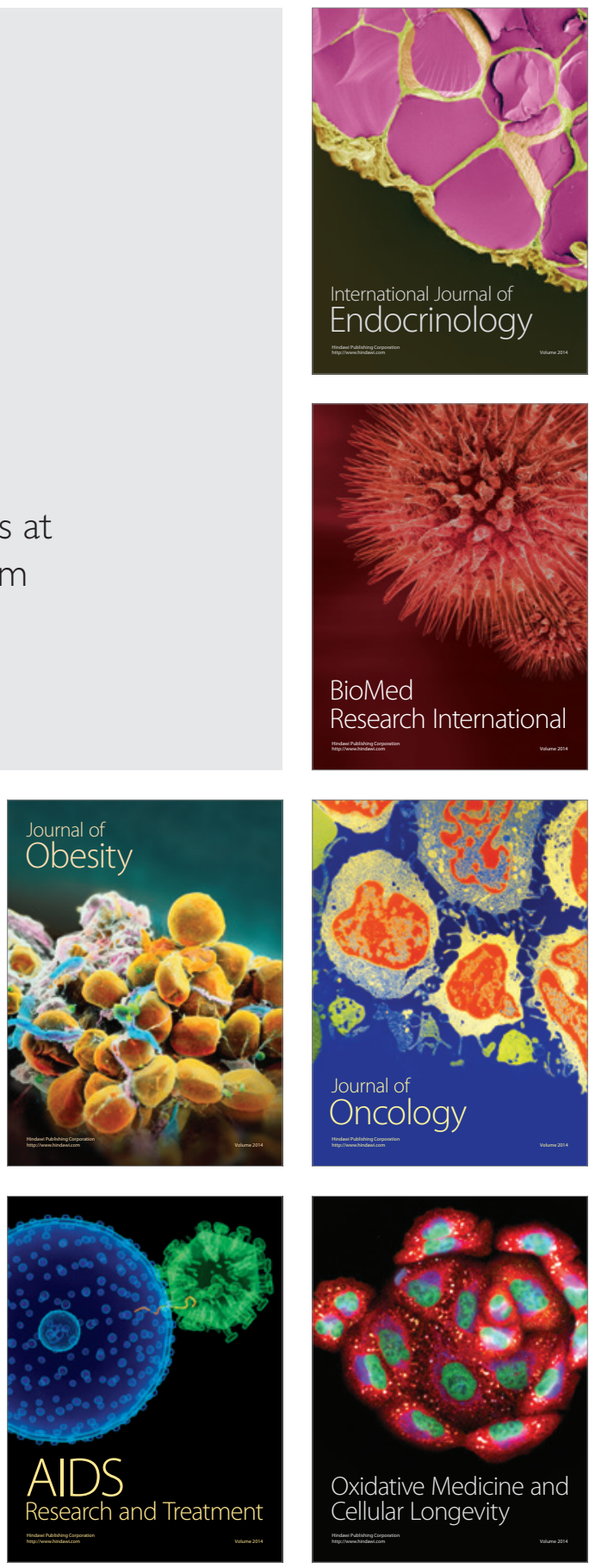